\title{
Multichannel Kondo Models in Non-Abelian Quantum Hall Droplets
}

\author{
Gregory A. Fiete, ${ }^{1}$ Waheb Bishara, ${ }^{1}$ and Chetan Nayak ${ }^{2,3}$ \\ ${ }^{1}$ Department of Physics, California Institute of Technology, MC 114-36, Pasadena, California 91125, USA \\ ${ }^{2}$ Microsoft Research, Station Q, CNSI Building, University of California, Santa Barbara, California 93106, USA \\ ${ }^{3}$ Department of Physics, University of California, Santa Barbara, California, 93106, USA
}

(Received 5 April 2008; published 20 October 2008)

\begin{abstract}
We study the coupling between a quantum dot and the edge of a non-Abelian fractional quantum Hall state which is spatially separated from it by an integer quantum Hall state. Near a resonance, the physics at energy scales below the level spacing of the edge states of the dot is governed by a $k$-channel Kondo model when the quantum Hall state is a Read-Rezayi state at filling fraction $\nu=2+k /(k+2)$ or its particle-hole conjugate at $\nu=2+2 /(k+2)$. The $k$-channel Kondo model is channel isotropic even without fine-tuning in the former state; in the latter, it is generically channel anisotropic. In the special case of $k=2$, our results provide a new venue, realized in a mesoscopic context, to distinguish between the Pfaffian and anti-Pfaffian states at filling fraction $\nu=5 / 2$.
\end{abstract}

DOI: 10.1103/PhysRevLett.101.176801

Non-Abelian quantum Hall states have sparked considerable interest recently because of their potential application to topological quantum computing [1]. Though it is not known whether such states exist, it is suspected that the observed plateaus at $\sigma_{x y}=\nu \frac{e^{2}}{h}$ with $\nu=5 / 2$ [2] and $\nu=$ $12 / 5$ [3] are due to non-Abelian quantum Hall states. The evidence, thus far, comes primarily from numerical studies [4-6] which found that the ground states of small numbers of electrons had large overlap with the Moore-Read Pfaffian wave function [7] and the particle-hole conjugate of the $k=3$ Read-Rezayi (RR) wave function [8,9] at $\nu=$ $5 / 2$ and $\nu=12 / 5$ respectively, and from recent noise and tunneling measurements $[10,11]$. It has been argued that these wave functions are representatives of two universality classes which exhibit non-Abelian quasiparticle statistics, which is a necessary ingredient for topological quantum computing [12]. Recently, further numerical studies [13] have bolstered the argument that these states occur in the experiments of Refs. [2,3].

Some theoretical proposals have been made to determine if the $\nu=5 / 2$ state possesses the non-Abelian quasiparticle statistics of the Pfaffian [14,15]. While fabricating high-mobility samples of mesoscopic size presents a significant challenge, recent experiments on quantum point contacts at $\nu=5 / 2$ give one reason to believe that such devices are within reach [16]. Experiments on such devices have recently shed light, for the first time, on quasiparticle properties at $\nu=5 / 2$. Shot noise [10] and nonlinear current-voltage characteristics [11] at quantum point contacts at $\nu=5 / 2$ are both consistent with a quasiparticle charge of $e / 4$, as required by the Moore-Read Pfaffian state.

However, the "anti-Pfaffian" is an equally good candidate at $\nu=5 / 2$ [17]. The anti-Pfaffian is the conjugate of the Pfaffian under particle-hole symmetry within a Landau level, which is an exact symmetry in the limit of large magnetic field. This symmetry must be spontaneously
PACS numbers: 73.43. $-\mathrm{f}, 71.10 . \mathrm{Pm}$ broken in order for one of these two degenerate ground states to occur; the system sizes studied in numerics on the torus were too small to observe anything other than the symmetric combination of the two [5]. For numerics on the sphere [4], the finite geometry explicitly breaks the symmetry; the anti-Pfaffian occurs at a different value of the magnetic flux and was, consequently, missed.

The Pfaffian and anti-Pfaffian states differ significantly in the nature of their edge excitations, and thus differ in tunneling characteristics and thermal transport along the edge $[17,18]$. Both states possess charge $e / 4$ quasiparticles, so the existing noise experiments do not allow one to distinguish between them [10]. Current-voltage characteristics at a point contact can distinguish between the two states; measurements appear to be more consistent with the anti-Pfaffian state although they cannot fully rule out the Pfaffian [11]. Therefore, there is urgent need for further experiments to determine not only whether the $\nu=5 / 2$ state is Abelian or non-Abelian, but to indicate which nonAbelian state. In this Letter, we propose such experiments and generalize our discussion of the Pfaffian state to cover the Read-Rezayi states as well. Our proposal is largely orthogonal to existing ones $[19,20]$.

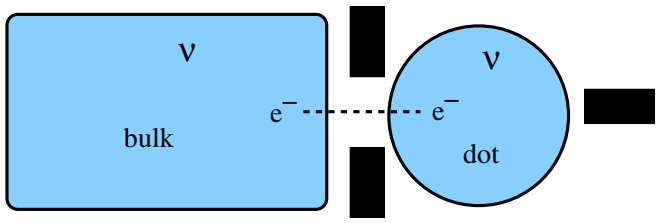

FIG. 1 (color online). Schematic of our model. Gates are shown in black. They may be used to form a point contact to pinch off the dot from the rest of the quantum Hall bulk. The gate on the right of the figure may be used to shift the energy levels of the dot by changing its area $S$. The bulk is assumed to be at filling fraction $\nu=2+k /(k+2)$ or $\nu=2+2 /(k+2)$. The white region between the dot and the bulk is assumed to be at $\nu=2$. The charge on the dot may be measured capacitively [22]. 
In this Letter, we study the setup shown in Fig. 1. The bulk quantum Hall state on the left is assumed to be in a non-Abelian fractional quantum Hall state at $\nu=$ $2+k /(k+2)$ or $\nu=2+2 /(k+2)$. A quantum point contact may be used to pinch off a finite region of the quantum Hall fluid and form a quantum dot separated from the bulk by a tunneling barrier. We assume that the lower two Landau levels are not pinched off and therefore do not backscatter at the point contact; the barrier region is assumed to have $\nu=2$. For a finite system the edge modes acquire a discrete spectrum $[19,21]$. We focus on fluctuations of the quantum dot charge $Q \equiv e\left\langle\hat{N}_{e}\right\rangle$ near a degeneracy point in the energy: $E\left(N_{e}, S, B\right)=E\left(N_{e}+1, S, B\right)$ where $N_{e}$ is the number of electrons. The energy $E$ depends on area $S$, which may be altered by a gate potential shown in Fig. 1, and on the magnetic field $B$. The charge of the dot can be measured capacitively [22].

We assume energy and temperature scales are much less than the level spacing of the dot edge states. Then only two levels on the quantum dot (the degenerate or nearly degenerate ones) are important. These two levels act as an effective, local spin- $1 / 2$ degree of freedom. The crucial feature of the Read-Rezayi states is the tunneling coupling of their edge modes to this effective spin degree of freedom, which can be mapped to the $k$-channel Kondo model. (Our analysis applies to an effective spin degree of freedom which accounts for the charge on the dot; the Read-Rezayi states and their particle-hole conjugates [23] are spin polarized, so there are no spin-flips in the quantum Hall edge states). The mapping allows us to exploit known results from the multichannel Kondo models.

A detuning from degeneracy maps to the coupling of an external magnetic field to the spin in the $k$-channel Kondo model. Thus, charge susceptibilities in our quantum dot setup can be obtained from magnetic susceptibility in the Kondo model. A remarkable feature of the scenario we discuss here is that the channel isotropic limit is automatically obtained for Read-Rezayi states without any finetuning. This feature follows from the form of the coupling of the edge states to the quantum dot degrees of freedom. On the other hand, for their particle-hole conjugates $[17,23]$, the generic case is channel anisotropic. Since the channel isotropic and channel anisotropic Kondo models are very different, one can exploit the thermodynamics of the multichannel Kondo model applied to the charge susceptibility to distinguish the Pfaffian from the antiPfaffian. This is one of our central results.

Pfaffian state.-We begin with the case of a quantum dot coupled to a bulk quantum Hall state in the Moore-Read Pfaffian state. The Hamiltonian is $H=H_{\text {edge }}+H_{\text {dot }}+H_{\text {tun }}$. Here and henceforth, we ignore the 2 filled lower Landau levels. This is justified by the sequence of modes pinched off in a point contact [10]. The edge theory for the Pfaffian state is the product of a free, charged chiral bosonic sector and a neutral Majorana sector. The edge Hamiltonian takes the form

$$
H_{\text {edge }}=\int d x\left(v_{c} \frac{(k+2) / k}{4 \pi}\left(\partial_{x} \varphi(x)\right)^{2}+i v_{n} \psi \partial_{x} \psi\right),
$$

Here, $k=2$ and $v_{n}<v_{c}$ is the velocity of the neutral mode(s). The Hamiltonian of the dot describes a two-level system which we can take to be "empty" or "occupied" (later to be mapped to "up" or "down") [24]. It thus has a fermionic character and we label the fermionic annihilation (creation) operator for this state $d\left(d^{\dagger}\right)$. Thus, $H_{\mathrm{dot}}=$ $\epsilon_{d} d^{\dagger} d$, where $\epsilon_{d}=0$ at the degeneracy point and $\epsilon_{d} \neq 0$ when one is tuned away from degeneracy. The tunneling Hamiltonian is

$$
H_{\text {tun }}=t\left(d^{\dagger} \Psi_{e}(0)+\Psi_{e}^{\dagger}(0) d\right)+V d^{\dagger} d \Psi_{e}^{\dagger}(0) \Psi_{e}(0),
$$

where $t$ is the tunneling amplitude to the dot; $x=0$ is the location of the point contact; $V$ is the Coulomb repulsion between the edge and the dot, and $\Psi_{e}\left(\Psi_{e}^{\dagger}\right)$ is the annihilation (creation) operator for the electron, $\Psi_{e}^{\dagger}=\psi e^{i 2 \varphi}$. We use the convention $\operatorname{dim}\left[e^{i \alpha \varphi}\right]=\nu \frac{\alpha^{2}}{2}$, so that $\operatorname{dim}\left[\Psi_{e}\right]=3 / 2$.

As a result of the scaling $\operatorname{dim}$ of $\Psi_{e}, t$ is naively irrelevant,

$$
\frac{d t}{d \ell}=-\frac{1}{2} t+\mathcal{O}(t V)+\mathcal{O}\left(t^{3}\right) .
$$

However, for $V$ sufficiently large, $t$ flows to the 2-channel Kondo fixed point, not to $t=0$. To see this, we apply a unitary transformation $U=e^{2 i d^{\dagger} d \varphi(0)}$ to $H$, which rotates $\varphi(0)$ out of the tunneling term. $H$ now takes the form

$$
\begin{aligned}
U H U^{\dagger}= & H_{\text {edge }}+H_{\text {dot }}+t \psi\left(d-d^{\dagger}\right) \\
& +\left(V-2 v_{c}\right) d^{\dagger} d \partial_{x} \varphi(0) .
\end{aligned}
$$

For $V-2 v_{c}$, this is a purely quadratic theory which can be solved exactly. Thus, $t$ is clearly relevant in this limit; it is actually relevant over a range of values of $V$. Note that only the Majorana combination $d-d^{\dagger}$ couples to the quantum Hall edge. This is precisely the same feature which leads to non-Fermi liquid behavior in the 2-channel Kondo problem [25]: the spectral function $\operatorname{Im}\left\langle d^{\dagger} d\right\rangle$ has both a $\delta$-function piece, coming from $d^{\dagger}+d$ and a Lorentzian piece coming from $d-d^{\dagger}$. The coupling of a quantum dot to an antiPfaffian quantum Hall state does not have this property.

To see the connection to the two-channel Kondo model, it is useful to represent the two-level system on the dot by a spin: $S^{\dagger}=d^{\dagger}, S^{-}=d$, and $S^{z}=d^{\dagger} d-1 / 2$ (up to suppressed Klein factors). We apply a unitary transformation $U=e^{i \alpha S^{z} \varphi(0)}$ to $H$ as before, but take $\alpha=2-\sqrt{2}$, to partially rotate $\varphi(0)$ in the tunneling term, giving

$$
\begin{aligned}
U H U^{\dagger}= & H_{\text {edge }}+\epsilon_{d} S^{z}+\left(V-v_{c} \alpha\right) S^{z} \partial_{x} \varphi(0) \\
& +t\left(\psi^{\dagger} e^{-i \sqrt{2} \varphi(0)} S^{\dagger}+\psi e^{i \sqrt{2} \varphi(0)} S^{-}\right) .
\end{aligned}
$$

We now compare this to the Kondo model:

$$
H_{\mathrm{imp}}=\lambda_{\perp}\left(J^{+}(0) S^{-}+J^{-}(0) S^{+}\right)+\lambda_{z} J^{z}(0) S^{z}+h S^{z},
$$

where $\vec{S}$ is the impurity spin; $\vec{J}(0)$ is conduction electron 
spin density at the impurity site; $\lambda_{\perp}, \lambda_{z}$ are the exchange couplings not assumed to be equal; and $h$ is the magnetic field. Retaining only the $s$-wave channel, we have a chiral one-dimensional problem in which the impurity is at the origin and the incoming and outgoing modes are rightmoving modes at $x<0$ and $x>0$, respectively. Affleck and Ludwig observed [26] that the Hamiltonian of the conduction electrons $H_{\text {cond }}$ in the $k$-channel Kondo model admits a conformal decomposition, $H_{\text {cond }}=H_{\mathrm{U}(1)}+$ $H_{\mathrm{SU}(2)_{k}}+H_{\mathrm{SU}(k)_{2}}$. This decomposition reflects the $\mathrm{SU}(2)_{k}$ Kac-Moody algebra satisfied by the spin density $J^{a}$, which we now exploit in the $k=2$ case and, later, in the general case. For $k=2$, we can express the $J^{a}$ in terms of a Majorana fermion, $\psi$, and a free boson $\varphi$ :

$$
J^{\dagger}=\sqrt{2} \psi e^{i \sqrt{2} \varphi}, \quad J^{-}=\sqrt{2} \psi e^{-i \sqrt{2} \varphi}, \quad J^{z}=\sqrt{2} \partial_{x} \varphi .
$$

The operators $\psi$ and $\varphi$ have a complicated, nonlocal relation to the original conduction electron operators, but they have the virtue of satisfying the $\mathrm{SU}(2)_{2}$ Kac-Moody algebra via (7).

Substituting (7) into (6), we see that our problem (5) maps onto the 2-channel Kondo model with anisotropic exchange if we identify $\lambda_{\perp}=t, \sqrt{2} \lambda_{z}=V-(2-\sqrt{2}) v_{c}$, and $h=\epsilon_{d}$. For $\lambda_{z}<0$, the Kondo model is ferromagnetic. In the ferromagnetic Kondo model, the coupling to the impurity is irrelevant, as we naively expected above (3). However, when $V$ is sufficiently large, $\lambda_{z}>0$, corresponding to the antiferromagnetic Kondo model. In this case, the Hamiltonian is controlled in the infrared by the exchange and channel isotropic antiferromagnetic spin-1/2 2-channel Kondo fixed point [27]. This fixed point is characterized by non-Fermi liquid correlations, including anomalous exponents for the temperature dependence of the impurity contribution to the specific heat and spin susceptibility, and the magnetic field dependence of the zero-temperature magnetization. The latter two translate to the charge susceptibility and charge of the quantum dot:

$$
\chi_{\text {charge }} \propto \ln \left(T_{K} / T\right), \quad \Delta Q \propto V_{G} \ln \left(k_{B} T_{K} / e^{*} V_{G}\right),
$$

where the Kondo temperature depends on nonuniversal values $v_{n}, t$ and is given by $T_{K} \propto \exp \left(-c_{1} v_{n} / t\right)$ with $c_{1}>$ 0 . Here, $\Delta Q=Q-e\left(N_{e}+\frac{1}{2}\right)$ is the charge on the dot measured relative to the average electron number at the degeneracy point of the energy. In the case $k=2$, which corresponds to the Pfaffian state, possibly realized at $\nu=$ $5 / 2$, there are logarithmic corrections: Ordinarily, finetuning would be required to realize channel isotropy in the Kondo model [28] but, the coupling between a quantum dot and the edge of a Pfaffian quantum Hall state automatically realizes the channel isotropic 2-channel Kondo model.

Anti-Pfaffian state.-The edge theory of the antiPfaffian state is [17]

$$
\mathcal{L}_{\overline{\mathrm{Pf}}}=\frac{2}{4 \pi} \partial_{x} \phi_{\rho}\left(\partial_{t}-v_{\rho} \partial_{x}\right) \phi_{\rho}+i \psi_{a}\left(-\partial_{t}-v_{\sigma} \partial_{x}\right) \psi_{a} .
$$

There is a charged boson $\phi_{\rho}$ and three counterpropagating Majorana fermions $\psi_{a}, a=1,2,3$. There are three different dimension- $3 / 2$ electron operators, $\psi_{a} e^{2 i \phi_{\rho}}$. The combination $\left(\psi_{1}-i \psi_{2}\right) e^{2 i \phi_{\rho}}$ is inherited from the electron operator of the $\nu=1$ integer quantum Hall state in which a Pfaffian state of holes forms. Thus, we expect it to have the largest tunneling amplitude. The other two electron operators are complicated charge- $e$ combinations of the $\nu=1$ electron operator and the electron operator of the Pfaffian state of holes. The tunneling Hamiltonian is (the repeated index $a$ is summed over)

$$
H_{\text {tun }}=\left(t_{a} \psi_{a} e^{-2 i \phi_{\rho}} d^{\dagger}+\text { H.c. }\right)+V d^{\dagger} d \partial_{x} \phi_{\rho} .
$$

Performing a unitary transformation as before to rotate out the $\phi_{\rho}$ dependence of the first term, we obtain $U H U^{\dagger}=$ $H_{\text {edge }}+H_{\text {dot }}+\tilde{H}_{\text {tun }}$, where

$$
\begin{aligned}
\tilde{H}_{\mathrm{tun}}= & \left(t_{a} \psi_{a} d^{\dagger}+\text { H.c. }\right)+\left(V-2 v_{c}\right) d^{\dagger} d \partial_{x} \phi_{\rho} \\
= & i \chi_{1}\left(\lambda_{1}\left(d^{\dagger}-d\right) / i+\lambda_{1}^{\prime}\left(d^{\dagger}+d\right)\right)+i \lambda_{2} \chi_{2}\left(d^{\dagger}+d\right) \\
& +\left(V-2 v_{c}\right) d^{\dagger} d \partial_{x} \phi_{\rho},
\end{aligned}
$$

where $\chi_{1}=u_{a} \psi_{a} / \sqrt{u^{2}}, \quad \chi_{2}=w_{a} \psi_{a} / \sqrt{w^{2}}, \quad u_{a}=\operatorname{Re} t_{a}$, $v_{a}=\operatorname{Im} t_{a}, \quad w_{a}=v_{a}-u_{a}\left(u \cdot v / u^{2}\right), \quad \lambda_{1}=\sqrt{u^{2}}, \quad \lambda_{2}=$ $\sqrt{w^{2}}, \lambda_{1}^{\prime}=u \cdot v / \sqrt{u^{2}}$, and $\left\{\chi_{1}, \chi_{2}\right\}=0$. Note that, for generic $t_{a} s$, both $\left(d^{\dagger}-d\right)$ and $\left(d^{\dagger}+d\right) / i$ couple to the edge modes, as in the one-channel Kondo model. This is in contrast to the Pfaffian case, in which only $\left(d^{\dagger}-d\right)$ couples to the edge modes, as in the two-channel Kondo model. At the Toulouse point, the one-channel Kondo model can be mapped to a form similar to (11) with $V=$ $2 v_{c}$. The charge susceptibility and charge of the quantum dot have the temperature and voltage dependence characteristic of a Fermi liquid:

$$
\chi_{\text {charge }} \propto \text { const, } \quad \Delta Q \propto V_{G} .
$$

Consequently, measurements of the behavior of the dot would distinguish the Pfaffian and anti-Pfaffian states.

Read-Rezayi state.-Now we analyze the Read-Rezayi state with filling $k /(k+2)$, generalizing our discussion above of the Pfaffian state, which is the $k=2$ case. The edge Hamiltonian takes the form $H_{\text {edge }}=H_{c}+H_{Z_{k}}$ with $H_{c}$ the same as the first term in Eq. (1). $H_{Z_{k}}$ can be written as a gauged $\mathrm{SU}(2)_{k}$ WZW model in which the U(1) subgroup has been gauged, thereby realizing an $\mathrm{SU}(2)_{k} / \mathrm{U}(1)$ coset with central charge $c=\frac{3 k}{k+2}-1=\frac{2 k-2}{k+2}$, but we will not need this representation here. The electron operator now takes the form: $\Psi_{e}^{\dagger}=\psi_{1} e^{i \frac{k+2}{k}} \varphi$, where $\psi_{1}$ is a parafermion field. Since $\operatorname{dim}\left[\psi_{1}\right]=1-1 / k, \operatorname{dim}\left[\Psi_{e}\right]=3 / 2$.

As before, we apply a unitary transformation $U=$ $e^{i \alpha S^{z} \varphi(0)}$ to $H$, which now takes the form 


$$
\begin{aligned}
U H U^{\dagger}= & H_{\mathrm{edge}}+H_{\mathrm{dot}}+\left(V-v_{c} \alpha\right) S^{z} \partial_{x} \varphi(0) \\
& +t\left(\psi_{\dagger}^{1} e^{-i \tilde{\alpha} \varphi(0)} S^{\dagger}+\psi_{1} e^{i \tilde{\alpha} \varphi(0)} S^{-}\right),
\end{aligned}
$$

where $\quad \tilde{\alpha} \equiv \frac{k+2}{k}-\alpha$. The choice $\alpha^{*}=\frac{k+2}{k}-\sqrt{\frac{k+2}{k}} \sqrt{\frac{2}{k}}$ makes the connection to the $k$-channel Kondo problem explicit because the $\mathrm{SU}(2)_{k}$ current operators can be represented in terms of the $\mathbb{Z}_{k}$ parafermions: $J^{\dagger}=\sqrt{k} \psi_{1} e^{i \beta \varphi}$, $J^{-}=\sqrt{k} \psi_{1}^{\dagger} e^{-i \beta \varphi}, J^{z}=\frac{k}{2} \beta \partial_{x} \varphi$, where $\beta=\sqrt{2(k+2)} / k$. Substituting these expressions into (6) we see that our problem (13) is equivalent to the $k$-channel Kondo problem if we identify $\lambda_{\perp}=t, \beta \lambda_{z}=V-v_{c} \alpha^{*}$, and $h=\epsilon_{d}$. For $V>v_{c} \alpha^{*}$, this is the antiferromagnetic Kondo problem, which has an intermediate coupling fixed point. Thus, we see that the Read-Rezayi states offer a novel scenario to realize the non-Fermi liquid behavior of the $k$-channel Kondo model, $\chi_{\text {charge }} \propto T^{-(k-2) /(k+2)}, \Delta Q \propto V_{G}^{2 / k}$ which would otherwise require an incredible amount of finetuning for $k \geq 3$. Moreover, observing the predicted nonFermi liquid behavior would provide strong evidence that the quantum Hall state is of the Read-Rezayi type. It can also be shown that the particle-hole conjugate of the ReadRezayi state at $\nu=2+2 /(k+2)$ generalizes the result obtained above for the anti-Pfaffian: the Kondo model realized is not channel isotropic [9].

Tunneling through a quantum dot.-We now consider the situation of bulk $\nu=2+k /(k+2)$ quantum Hall states on either side of a quantum dot. By the arguments above, if the two tunneling amplitudes $t_{L}$ and $t_{R}$ are equal [29] then this model can be mapped onto the $2 k$-channel Kondo model. Consequently, the conductance through the dot is finite at zero-temperature, $G=G_{0}-a T^{2 /(k+2)}$ where $G_{0}, a$ are constants [30].

In summary, we have shown that a quantum dot coupled via tunneling to a Pfaffian quantum Hall state realizes the channel isotropic 2-channel Kondo model while a quantum dot coupled to a Read-Rezayi state of filling factor $\nu=$ $2+k /(k+2)$ leads to a channel isotropic $k$-channel Kondo problem, both without any fine-tuning of parameters. These systems will thus exhibit all the known nonFermi liquid properties in their thermodynamics, expressed through the charge on the dot, which may be measured capacitively. Because the coupling of a quantum dot to an anti-Pfaffian state generically exhibits Fermi liquid properties our results may be used to distinguish between the two leading candidate states for $\nu=5 / 2$, the Pfaffian and the anti-Pfaffian.

We acknowledge helpful discussions with E. Ardonne, J. Eisenstein, L. Fidkowski, A. Ludwig, and G. Refael. G. A.F. was supported by the Lee A. DuBridge Foundation.

[1] S. Das Sarma, M. Freedman, and C. Nayak, Phys. Today 59, No. 7, 32 (2006).
[2] R. Willett et al., Phys. Rev. Lett. 59, 1776 (1987); J.P. Eisenstein et al., ibid. 88, 076801 (2002); W. Pan et al., ibid. 83, 3530 (1999); H. Choi et al., Phys. Rev. B 77, 081301 (2008).

[3] J. S. Xia et al., Phys. Rev. Lett. 93, 176809 (2004).

[4] R. H. Morf, Phys. Rev. Lett. 80, 1505 (1998).

[5] E. H. Rezayi and F. D. M. Haldane, Phys. Rev. Lett. 84, 4685 (2000).

[6] E. H. Rezayi and N. Read, arXiv:cond-mat/0608346.

[7] G. Moore and N. Read, Nucl. Phys. B360, 362 (1991); M. Greiter, X. G. Wen, and F. Wilczek, Nucl. Phys. B374, 567 (1992); C. Nayak and F. Wilczek, Nucl. Phys. V479, 529 (1996).

[8] N. Read and E. Rezayi, Phys. Rev. B 59, 8084 (1999).

[9] G. Fiete, W. Bishara, and C. Nayak (to be published).

[10] M. Dolev et al., Nature (London) 452, 829 (2008).

[11] I. P. Radu et al., Science 320, 899 (2008).

[12] C. Nayak et al., Rev. Mod. Phys. 80, 1083 (2008).

[13] A. Feiguin et al., Phys. Rev. Lett. 100, 166803 (2008); M. R. Peterson, Th. Jolicoeur, and S. Das Sarma, Phys. Rev. B 78, 155308 (2008).

[14] E. Fradkin et al., Nucl. Phys. B516, 704 (1998); S. Das Sarma, M. Freedman, and C. Nayak, Phys. Rev. Lett. 94, 166802 (2005); A. Stern and B. I. Halperin, Phys. Rev. Lett. 96, 016802 (2006); I. Affleck and A. W. W. Ludwig, Nucl. Phys. B360, 641 (1991).

[15] P. Bonderson, A. Kitaev, and K. Shtengel, Phys. Rev. Lett. 96, 016803 (2006).

[16] J. B. Miller et al., Nature Phys. 3, 561 (2007).

[17] M. Levin, B. I. Halperin, and B. Rosenow, Phys. Rev. Lett. 99, 236806 (2007); S.-S. Lee et al., ibid. 99, 236807 (2007).

[18] W. Bishara and C. Nayak, Phys. Rev. B 77, 165302 (2008).

[19] R. Ilan, E. Grosfeld, and A. Stern, Phys. Rev. Lett. 100, 086803 (2008).

[20] P. Bonderson et al., Phys. Rev. Lett. 97, 016401 (2006); D. Feldman et al., Phys. Rev. B 76, 085333 (2007); L. Fidkowski, arXiv:0704.3291; K. T. Law, Phys. Rev. B 77, 205310 (2008).

[21] G. A. Fiete, G. Refael, and M. P. A. Fisher, Phys. Rev. Lett. 99, 166805 (2007).

[22] D. Berman et al., Phys. Rev. Lett. 82, 161 (1999).

[23] W. Bishara, G. A. Fiete, and C. Nayak, Phys. Rev. B 77, 241306 (2008).

[24] A. Furusaki and K. A. Matveev, Phys. Rev. Lett. 88, 226404 (2002); E. H. Kim et al., J. Phys.: Condens. Matter 15, 7047 (2003); K. A. Matveev, Phys. Rev. B 51, 1743 (1995).

[25] V. J. Emery and S. Kivelson, Phys. Rev. B 46, 10812 (1992).

[26] I. Affleck, Nucl. Phys. B336, 517 (1990); I. Affleck and A. W. W. Ludwig, Nucl. Phys. B352, 849 (1991).

[27] I. Affleck et al., Phys. Rev. B 45, 7918 (1992).

[28] Y. Oreg and D. Goldhaber-Gordon, Phys. Rev. Lett. 90, 136602 (2003).

[29] C. Kane and M.P. A. Fisher, Phys. Rev. B 46, 15233 (1992).

[30] A. W. W. Ludwig and I. Affleck, Phys. Rev. Lett. 67, 3160 (1991); I. Affleck and A. W. W. Ludwig, Phys. Rev. B 48, 7297 (1993). 